\title{
CONVERGENCE IN VARIABLE LEBESGUE SPACES
}

\author{
David Cruz-Uribe, SFO and Alberto Fiorenza
}

\begin{abstract}
We consider the relationship in the variable Lebesgue space $L^{p(\cdot)}(\Omega)$ between convergence in norm, convergence in modular, and convergence in measure, for both bounded and unbounded exponent functions.
\end{abstract}

\section{Introduction}

The variable Lebesgue spaces are a generalization of the classical Lebesgue spaces, where the constant exponent $p$ is replaced by a function $p(\cdot)$. The variable Lebesgue spaces have a long history, having first been considered by Orlicz [4] in the 1930's. They have been the subject of renewed investigation for the past 15 years, both for their own intrinsic interest and for their applications to the calculus of variations and to problems in physics. For more information we refer the reader to the surveys by Diening, Hästö and Nekvinda [1] and Samko [6].

In this paper we study the relationship between convergence in measure, convergence in norm and convergence in modular in variable Lebesgue spaces. To state our results, we must first give some basic definitions. For complete information on the properties of variable Lebesgue spaces, see $[\mathbf{3}]$ (also see $[\mathbf{2}]$ ).

Given a measurable set $\Omega \subset \mathbb{R}^{n}$, an exponent function is a measurable function $p(\cdot): \Omega \rightarrow[1, \infty]$. We denote the set of all such functions by $\mathcal{P}(\Omega)$. Given $p(\cdot) \in \mathcal{P}(\Omega)$, let $\Omega_{\infty}=\{x \in \Omega: p(x)=\infty\}$, and for any $E \subset \Omega$, let

$$
p_{-}(E)=\underset{x \in E}{\operatorname{essinf}} p(x), \quad p_{+}(E)=\underset{x \in E}{\operatorname{ess} \sup } p(x) .
$$

For brevity, we will let $p_{-}=p_{-}(\Omega)$ and $p_{+}=p_{+}(\Omega)$.

2000 Mathematics Subject Classification. 42B25, 42B35.

Key words. Variable Lebesgue space, norm convergence, modular convergence, convergence in measure.

The first author is supported by the Stewart-Dorwart faculty development fund at Trinity College. 
We define the modular functional

$$
\rho(f)=\int_{\Omega \backslash \Omega_{\infty}}|f(x)|^{p(x)} d x+\|f\|_{L^{\infty}\left(\Omega_{\infty}\right)} .
$$

If $\left|\Omega_{\infty}\right|=0$, then we set the last term equal to 0 ; if $\left|\Omega \backslash \Omega_{\infty}\right|=0$, then $\rho(f)=\|f\|_{L^{\infty}\left(\Omega_{\infty}\right)}$. Given $E \subset \Omega$, let $\rho_{E}(f)=\rho\left(f \chi_{E}\right)$. We define the space $L^{p(\cdot)}(\Omega)$ to be the set of measurable functions such that for some $\lambda>0, \rho(f / \lambda)<\infty$. This is a Banach function space when equipped with the norm

$$
\|f\|_{p(\cdot)}=\inf \{\lambda>0: \rho(f / \lambda) \leq 1\} .
$$

When $p(\cdot)=p$, a constant, then $L^{p(\cdot)}(\Omega)=L^{p}(\Omega)$ with equality of norms.

We consider three kinds of convergence on variable Lebesgue spaces: convergence in norm, convergence in modular, and convergence in measure. Given a sequence $\left\{f_{k}\right\} \subset L^{p(\cdot)}(\Omega)$, we say that it converges in norm to $f \in L^{p(\cdot)}(\Omega)$ if $\left\|f-f_{k}\right\|_{p(\cdot)} \rightarrow 0$ as $k \rightarrow \infty$. We say that $f_{k} \rightarrow f$ in modular if there exists $\beta>0$ such that $\rho\left(\beta\left(f-f_{k}\right)\right) \rightarrow 0$ as $k \rightarrow \infty$. And we recall the definition of convergence in measure: given any $\epsilon>0$, then for all $k$ sufficiently large

$$
\left|\left\{x \in \Omega:\left|f(x)-f_{k}(x)\right| \geq \epsilon\right\}\right|<\epsilon .
$$

Our starting point for studying the relationship between these three types of convergence is a result due to Fan and Zhao [2].

Theorem 1.1. Given $\Omega$ and $p(\cdot) \in \mathcal{P}(\Omega)$, suppose $p_{+}<\infty$. Then given $f \in L^{p(\cdot)}(\Omega)$ and a sequence $\left\{f_{k}\right\} \subset L^{p(\cdot)}(\Omega)$, the following are equivalent:

(1) $f_{k} \rightarrow f$ in norm,

(2) $f_{k} \rightarrow f$ in modular,

(3) $f_{k} \rightarrow f$ in measure and for some $\gamma>0, \rho\left(\gamma f_{k}\right) \rightarrow \rho(\gamma f)$.

Their proof, however, was only sketched, with many technical details omitted. The equivalence of (1) and (2) was proved in [3]; we will prove this equivalence in a more general setting in Theorem 1.3 below. In Section 2 below we give a proof of the equivalence of (2) and (3); we do so both for completeness and because it provides a model for the proofs of our other results.

Remark 1.2. If $f_{k} \rightarrow f$ in norm or modular, then we can strengthen our conclusion slightly. In the proof we actually show that $\rho\left(f_{k}\right) \rightarrow \rho(f)$. 
Since if $f_{k} \rightarrow f$ in measure, $\gamma f_{k} \rightarrow \gamma f$ in measure for any $\gamma>0$, and we can actually conclude that $\rho\left(\gamma f_{k}\right) \rightarrow \rho(\gamma f)$ for any $\gamma>0$.

Our primary goal in this paper is to examine each of these equivalences for all possible $p(\cdot) \in \mathcal{P}(\Omega)$, including the case $p_{+}=\infty$. In this case the situation is much more complicated and so before stating our precise results we want to briefly describe what happens to each implication in Theorem 1.1:

- In general, norm convergence always implies convergence in both modular and measure.

- If $p(\cdot)$ is bounded on $\Omega \backslash \Omega_{\infty}$ or if $p(\cdot)=\infty$, then modular convergence implies norm convergence.

- Convergence in measure, even with the additional hypothesis $\rho\left(\gamma f_{k}\right) \rightarrow \rho(\gamma f)$ used in Theorem 1.1, implies norm convergence only if $p_{+}<\infty$.

- Besides the case considered in Theorem 1.1, modular convergence implies convergence in measure exactly when the set $\{x \in \Omega$ : $p(x)>M\}$ gets small as $M$ gets large.

- Convergence in measure implies modular convergence if we assume $\left|\Omega_{\infty}\right|=0$ and if we replace the hypothesis $\rho\left(\gamma f_{k}\right) \rightarrow \rho(\gamma f)$ used above with a slightly stronger one.

We prove these results in Theorems 1.3, 1.4, 1.5, and 1.8; the contents of each theorem are summarized in the following diagram.

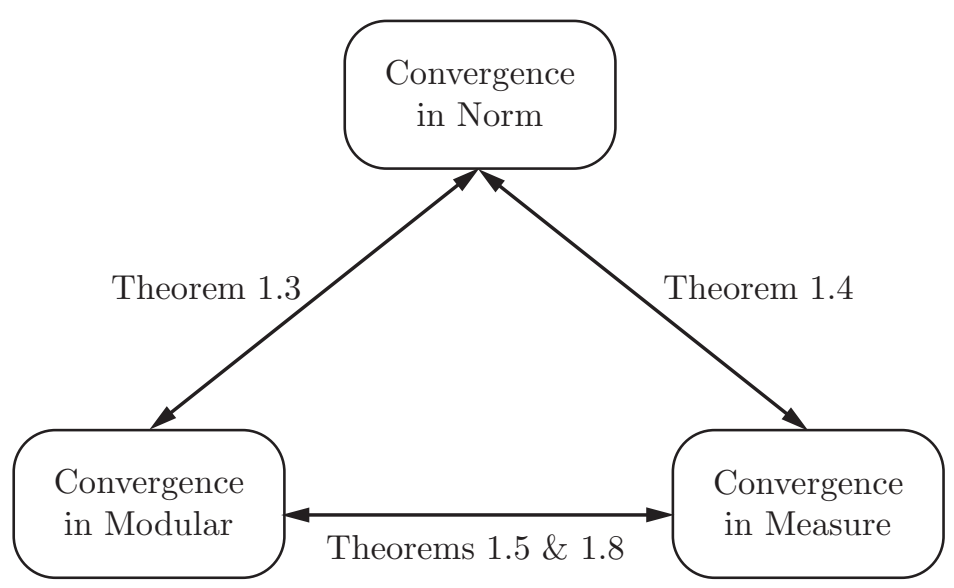

We now turn to the precise statement of our results. We first consider the relationship between norm and modular convergence. 
Theorem 1.3. Given $\Omega$ and $p(\cdot) \in \mathcal{P}(\Omega)$, and given a sequence $\left\{f_{k}\right\} \subset$ $L^{p(\cdot)}(\Omega)$ and $f \in L^{p(\cdot)}(\Omega)$, if $f_{k} \rightarrow f$ in norm then $f_{k} \rightarrow f$ in modular. Conversely, if the sequence converges in modular, then it converges in norm if and only if either $p_{-}=\infty$ or $p_{+}\left(\Omega \backslash \Omega_{\infty}\right)<\infty$.

Theorem 1.3 is essentially proved in [3] but their hypotheses for the second half are stated incorrectly. They assume $p_{+}<\infty$ but only use the weaker condition $p_{+}\left(\Omega \backslash \Omega_{\infty}\right)<\infty$. For completeness we will give their proof of Theorem 1.3, with the necessary modifications, in Section 3.

The relationship between convergence in norm and convergence in measure is equally straightforward.

Theorem 1.4. Given $\Omega$ and $p(\cdot) \in \mathcal{P}(\Omega)$, and given a sequence $\left\{f_{k}\right\} \in$ $L^{p(\cdot)}(\Omega)$ and $f \in L^{p(\cdot)}(\Omega)$, if $f_{k} \rightarrow f$ in norm, then $f_{k} \rightarrow f$ in measure and for all $\gamma>0$ sufficiently small, $\rho\left(\gamma f_{k}\right) \rightarrow \rho(\gamma f)$. If $p_{+}=\infty$, then the converse is false: convergence in measure and $\rho\left(\gamma f_{k}\right) \rightarrow \rho(\gamma f)$ for some $\gamma>0$ do not imply convergence in norm.

We will prove Theorem 1.4 in Section 4 below.

The relationship between modular convergence and convergence in measure is more complicated. If $p_{+}\left(\Omega \backslash \Omega_{\infty}\right)<\infty$, then we can immediately modify the proof of Theorem 1.1 -replacing $p_{+}$with $p_{+}\left(\Omega \backslash \Omega_{\infty}\right)$ and using the fact that on $\Omega_{\infty}$ the modular is just the $L^{\infty}$ norm - to prove that convergence in modular implies convergence in measure. (Details are left to the reader.) Trivially, the same is true if $p_{-}=\infty$.

If $p_{+}\left(\Omega \backslash \Omega_{\infty}\right)=\infty$, then convergence in modular need not imply convergence in measure. For example, let $\Omega=(1, \infty), p(x)=x$, and $f_{k}=\frac{1}{2} \chi_{(k, k+1)}$. Then $\rho\left(f_{k}\right) \leq 2^{-k}$, and so $\rho\left(f_{k}\right) \rightarrow 0$. However, for all $\epsilon<1 / 2,\left|\left\{x \in(1, \infty):\left|f_{k}(x)\right| \geq \epsilon\right\}\right|=1$, so the sequence does not converge to 0 in measure.

However, this implication does not always fail: rather, it depends on the size of the set where $p(\cdot)$ is unbounded. More precisely, we have the following result.

Theorem 1.5. Given $\Omega$ and $p(\cdot) \in \mathcal{P}(\Omega)$, for each $M \geq 1$ let

$$
G_{M}=\left\{x \in \Omega \backslash \Omega_{\infty}: p(x)>M\right\} .
$$

Then the following are equivalent:

(1) for any sequence $\left\{f_{k}\right\} \in L^{p(\cdot)}(\Omega)$ and $f \in L^{p(\cdot)}(\Omega)$, if $f_{k} \rightarrow f$ in modular, then $f_{k} \rightarrow f$ in measure and for every $\gamma>0$ sufficiently small, $\rho\left(\gamma f_{k}\right) \rightarrow \rho(\gamma f)$;

(2) $\left|G_{M}\right| \rightarrow 0$ as $M \rightarrow \infty$. 
Remark 1.6. Condition (2) holds automatically if $\left|\Omega \backslash \Omega_{\infty}\right|<\infty$, so in this case modular convergence always implies convergence in measure. This is analogous to the fact that pointwise convergence implies convergence in measure when $|\Omega|<\infty$.

We will prove Theorem 1.5 in Section 5 below. The proof is gotten by adapting the corresponding part of the proof of Theorem 1.1.

The reverse implication, that convergence in measure implies convergence in modular, can also fail to hold if $p(\cdot)$ is unbounded. However, it fails in different circumstances. It is never true if $\left|\Omega_{\infty}\right|>0$ : we will show that convergence in measure is always strictly weaker than convergence in modular. In the classical case convergence in measure does not imply convergence in the $L^{\infty}$ norm. The same example works in our case; we include it for completeness.

Example 1.7. Given $\Omega$ and $p(\cdot) \in \mathcal{P}(\Omega)$, suppose $\left|\Omega_{\infty}\right|>0$. Fix a sequence $\left\{E_{k}\right\}$ of subsets of $\Omega_{\infty}$ with positive measure such that $\left|E_{k}\right| \rightarrow$ 0 . Let $f=\chi_{\Omega_{\infty}}$ and for each $k$ define

$$
f_{k}= \begin{cases}1 & x \in \Omega_{\infty} \backslash E_{k} \\ -1 & x \in E_{k} .\end{cases}
$$

Then for all $k, \rho(f)=\|f\|_{L^{\infty}\left(\Omega_{\infty}\right)}=1=\left\|f_{k}\right\|_{L^{\infty}\left(\Omega_{\infty}\right)}=\rho\left(f_{k}\right)$, and $\left|\left\{x \in \Omega_{\infty}:\left|f(x)-f_{k}(x)\right|>0\right\}\right|=\left|E_{k}\right|$, so $f_{k} \rightarrow f$ in measure. However, for any $\beta>0$ and any $k, \rho\left(\beta\left(f-f_{k}\right)\right)=\beta\left\|f-f_{k}\right\|_{L^{\infty}\left(\Omega_{\infty}\right)}=2 \beta>0$, so the sequence does not converge in modular.

If $\left|\Omega_{\infty}\right|=0$, then we can show that convergence in measure implies convergence in modular provided that we slightly strengthen the hypothesis that $\rho\left(\gamma f_{k}\right) \rightarrow \rho(\gamma f)$ for some $\gamma>0$.

Theorem 1.8. Given $\Omega$ and $p(\cdot) \in \mathcal{P}(\Omega)$ such that $\left|\Omega_{\infty}\right|=0$, if $f \in$ $L^{p(\cdot)}(\Omega)$ and $\left\{f_{k}\right\} \subset L^{p(\cdot)}(\Omega)$ are such that $f_{k} \rightarrow f$ in measure and for some $\gamma, 0<\gamma<1, \rho(\gamma f)<\infty$ and $\rho\left(\gamma f_{k} / 3\right) \rightarrow \rho(\gamma f / 3)$, then $f_{k} \rightarrow f$ in modular.

We prove Theorem 1.8 in Section 6 below.

We can replace the value " 3 " in the hypothesis with any $r>2$; this follows immediately from the proof. This hypothesis, though stronger than that in Theorem 1.1, is not unnatural since as we noted above, Theorems 1.1 and 1.5 actually show that convergence in modular implies that for all $\gamma$ sufficiently small, $\rho\left(\gamma f_{k}\right) \rightarrow \rho(\gamma f)$. We conjecture, but 
cannot prove, that if $|\Omega|<\infty$, and this limit holds for some $\gamma_{0}>0$, then it is true for all $\gamma<\gamma_{0}$. We are not certain what is true if $|\Omega|=\infty$.

Finally, we show that the hypothesis $\rho\left(\gamma f_{k} / 3\right) \rightarrow \rho(\gamma f / 3)$ is necessary to get convergence in measure.

Example 1.9. Given $\Omega$ and $p(\cdot) \in \mathcal{P}(\Omega)$ such that $\left|\Omega_{\infty}\right|=0$, there exists a sequence of functions $f_{k} \in L^{p(\cdot)}(\Omega)$ and $f \in L^{p(\cdot)}(\Omega)$ such that $f_{k} \rightarrow f$ in measure, but for any $\gamma, 0<\gamma<1,\left\{\rho\left(\gamma f_{k}\right)\right\}$ does not converge to $\rho(\gamma f)$, and $\left\{f_{k}\right\}$ does not converge to $f$ in modular.

The construction of Example 1.9 is given at the end of Section 6 .

\section{Proof of Theorem 1.1}

The equivalence of (1) and (2) if $p_{+}<\infty$ is a special case of Theorem 1.3 and is proved in Section 3 below. Here we will prove that (2) and (3) are equivalent. We will first prove that (2) implies (3) and then prove the converse. Our proof is based on the proof sketched in [2].

Suppose that $f_{k} \rightarrow f$ in modular. Then there exists $\beta>0$ such that $\rho\left(\beta\left(f-f_{k}\right)\right) \rightarrow 0$ as $k \rightarrow \infty$. But since $p_{+}<\infty$, it is immediate from the definition of the modular that for any $\alpha>0$,

$$
\rho\left(\alpha\left(f-f_{k}\right)\right) \leq(1+\alpha / \beta)^{p_{+}} \rho\left(\beta\left(f-f_{k}\right)\right),
$$

and so $\rho\left(\alpha\left(f-f_{k}\right)\right) \rightarrow 0$. Take $\alpha=1$. Then $\rho\left(f-f_{k}\right) \rightarrow 0$; hence, $\left|f(\cdot)-f_{k}(\cdot)\right|^{p(\cdot)} \rightarrow 0$ in $L^{1}(\Omega)$ norm and so in measure. But then for any $\epsilon, 0<\epsilon<1$,

$\left|\left\{x \in \Omega:\left|f(x)-f_{k}(x)\right| \geq \epsilon\right\}\right| \leq\left|\left\{x \in \Omega:\left|f(x)-f_{k}(x)\right|^{p(x)} \geq \epsilon^{p_{+}}\right\}\right|<\epsilon^{p_{+}}<\epsilon$.

Hence $f_{k} \rightarrow f$ in measure.

We will now show that convergence in modular implies that $\rho\left(\gamma f_{k}\right) \rightarrow$ $\rho(\gamma f)$ for $\gamma=1$. We begin with an elementary inequality. By the mean value theorem, if $1 \leq p<\infty$ and $a, b \geq 0$, then

$$
\left|a^{p}-b^{p}\right| \leq p \max \left(a^{p-1}, b^{p-1}\right)|a-b| \leq p\left(a^{p-1}+b^{p-1}\right)|a-b| .
$$

Therefore,

$$
\begin{aligned}
\left|\rho(f)-\rho\left(f_{k}\right)\right| & \leq\left.\int_{\Omega}|| f(x)\right|^{p(x)}-\left|f_{k}(x)\right|^{p(x)} \mid d x \\
& \leq p_{+} \int_{\Omega}\left(|f(x)|^{p(x)-1}+\left|f_{k}(x)\right|^{p(x)-1}\right)\left|f(x)-f_{k}(x)\right| d x .
\end{aligned}
$$


To estimate the right-hand side we write the domain of integration as $\Omega_{1} \cup \Omega_{*}$, where $\Omega_{1}=\{x \in \Omega: p(x)=1\}$ and $\Omega_{*}=\{x \in \Omega: 1<p(x)<$ $\infty\}$. The integral on $\Omega_{1}$ is straightforward to estimate:

$$
\begin{aligned}
p_{+} \int_{\Omega_{1}}\left(|f(x)|^{p(x)-1}\right. & \left.+\left|f_{k}(x)\right|^{p(x)-1}\right)\left|f(x)-f_{k}(x)\right| d x \\
& =2 p_{+} \int_{\Omega_{1}}\left|f(x)-f_{k}(x)\right|^{p(x)} d x \leq 2 p_{+} \rho\left(f-f_{k}\right) .
\end{aligned}
$$

As we noted above, the right-hand side tends to 0 as $k \rightarrow \infty$.

To estimate the integral on $\Omega_{*}$, fix $\epsilon, 0<\epsilon<1 / 4$, and apply Young's inequality to get

$$
\begin{aligned}
& p_{+} \int_{\Omega_{*}}\left(|f(x)|^{p(x)-1}+\left|f_{k}(x)\right|^{p(x)-1}\right)\left|f(x)-f_{k}(x)\right| d x \\
\leq & p_{+} \int_{\Omega_{*}} \frac{\epsilon^{p^{\prime}(x)}}{p^{\prime}(x)}\left(|f(x)|^{p(x)-1}+\left|f_{k}(x)\right|^{p(x)-1}\right)^{p^{\prime}(x)} d x \\
& +p_{+} \int_{\Omega_{*}} \frac{\epsilon^{-p(x)}}{p(x)}\left|f(x)-f_{k}(x)\right|^{p(x)} d x \\
= & I_{1}+I_{2} .
\end{aligned}
$$

We estimate $I_{1}$ and $I_{2}$ separately. Since $p(x)>1$ for all $x \in \Omega_{*}$,

$$
I_{2} \leq p_{+} \rho\left(\epsilon^{-1}\left(f-f_{k}\right)\right) .
$$

To estimate $I_{1}$ we need two additional inequalities: for $p>0$ and $a, b>0$, we have by elementary calculus that

$$
\begin{aligned}
& a^{p}+b^{p} \leq \max \left(1,2^{1-p}\right)(a+b)^{p}, \\
& (a+b)^{p} \leq \max \left(1,2^{p-1}\right)\left(a^{p}+b^{p}\right) .
\end{aligned}
$$

Hence, since $1<p^{\prime}(x)<\infty$ on $\Omega_{*}$,

$$
\begin{aligned}
I_{1} & \leq p_{+} \int_{\Omega_{*}} \epsilon^{p^{\prime}(x)} \max \left(1,2^{2-p(x)}\right)^{p^{\prime}(x)}\left(|f(x)|+\left|f_{k}(x)\right|\right)^{p(x)} d x \\
& \leq p_{+} \int_{\Omega_{*}}(4 \epsilon)^{p^{\prime}(x)}\left(2|f(x)|+\left|f(x)-f_{k}(x)\right|\right)^{p(x)} d x \\
& \leq 4 p_{+} \epsilon \int_{\Omega_{*}} 2^{p(x)-1}\left(2^{p(x)}|f(x)|^{p(x)}+\left|f(x)-f_{k}(x)\right|^{p(x)}\right) d x \\
& \leq p_{+} 2^{2 p_{+}+1} \epsilon \rho(f)+p_{+} 2^{p_{+}+1} \epsilon \rho\left(f-f_{k}\right) .
\end{aligned}
$$


Combining this with the previous estimate, we see that

$$
\begin{aligned}
p_{+} \int_{\Omega_{*}} & \left(|f(x)|^{p(x)-1}+\left|f_{k}(x)\right|^{p(x)-1}\right)\left|f(x)-f_{k}(x)\right| d x \\
& \leq p_{+} 2^{2 p_{+}+1} \epsilon \rho(f)+p_{+} 2^{p_{+}+1} \epsilon \rho\left(f-f_{k}\right)+p_{+} \rho\left(\epsilon^{-1}\left(f-f_{k}\right)\right) .
\end{aligned}
$$

Therefore, in the limit,

$$
\begin{aligned}
\limsup _{k \rightarrow \infty} p_{+} \int_{\Omega_{*}}\left(|f(x)|^{p(x)-1}+\left|f_{k}(x)\right|^{p(x)-1}\right) \mid f(x) & -f_{k}(x) \mid d x \\
& \leq p_{+} 2^{2 p_{+}+1} \epsilon \rho(f) .
\end{aligned}
$$

Since $\epsilon>0$ was arbitrary, we conclude that $\left|\rho(f)-\rho\left(f_{k}\right)\right| \rightarrow 0$.

Now suppose that $f_{k} \rightarrow f$ in measure and that for some $\gamma>0$, $\rho\left(\gamma f_{k}\right) \rightarrow \rho(\gamma f)$. Since we also have that $\gamma f_{k} \rightarrow \gamma f$ in measure, we may assume without loss of generality that $\gamma=1$. Then for each $\epsilon, 0<\epsilon<1$,

$$
\begin{aligned}
\left|\left\{x \in \Omega:\left|f(x)-f_{k}(x)\right|^{p(x)}>\epsilon\right\}\right| & \leq\left|\left\{x \in \Omega:\left|f(x)-f_{k}(x)\right|>\epsilon^{1 / p_{-}}\right\}\right| \\
& \leq\left|\left\{x \in \Omega:\left|f(x)-f_{k}(x)\right|>\epsilon\right\}\right| \leq \epsilon .
\end{aligned}
$$

Hence, $\left|f(\cdot)-f_{k}(\cdot)\right|^{p(\cdot)} \rightarrow 0$ in measure.

Further, arguing as we did above using inequalities (2.1) and (2.2), we have that

$$
\begin{aligned}
& \left.|| f(x)\right|^{p(x)}-\left|f_{k}(x)\right|^{p(x)} \mid \\
& \leq p_{+}\left(|f(x)|^{p(x)-1}+\left|f_{k}(x)\right|^{p(x)-1}\right)\left|f(x)-f_{k}(x)\right| \\
& \leq p_{+}|f(x)|^{p(x)-1}\left|f(x)-f_{k}(x)\right| \\
& \quad+p_{+} \max \left(1,2^{p(x)-2}\right)\left(|f(x)|^{p(x)-1}+\left|f(x)-f_{k}(x)\right|^{p(x)-1}\right)\left|f(x)-f_{k}(x)\right| \\
& \leq p_{+}\left(2^{p_{+}}+1\right)|f(x)|^{p(x)-1}\left|f(x)-f_{k}(x)\right|+p_{+} 2^{p_{+}}\left|f(x)-f_{k}(x)\right|^{p(x)} .
\end{aligned}
$$

Now fix $\epsilon, 0<\epsilon<1$. Since $|f(\cdot)|^{p(\cdot)} \in L^{1}(\Omega)$, there exists $M \geq 1$ such that

$$
\left|\left\{x:|f(x)|^{p(x)-1}>M\right\}\right| \leq\left|\left\{x:|f(x)|^{p(x)}>M\right\}\right| \leq \epsilon / 2 .
$$


By inequality (2.3), since $f_{k} \rightarrow f$ and $\left|f(\cdot)-f_{k}(\cdot)\right|^{p(\cdot)} \rightarrow 0$ in measure, for all $k$ sufficiently large,

$$
\begin{aligned}
\mid\left\{x:\left.|| f(x)\right|^{p(x)}-\mid f_{k}(x)\right. & \left.\left.\right|^{p(x)} \mid>\epsilon\right\} \mid \\
\leq & \left|\left\{x:|f(x)|^{p(x)-1}>M\right\}\right| \\
& +\left|\left\{x: p_{+}\left(2^{p_{+}}+1\right) M\left|f(x)-f_{k}(x)\right|>\epsilon / 2\right\}\right| \\
& +\left|\left\{x: p_{+} 2^{p_{+}}\left|f(x)-f_{k}(x)\right|^{p(x)}>\epsilon / 2\right\}\right| \\
& <\frac{\epsilon}{2}+\frac{\epsilon}{2 p_{+}\left(2^{p_{+}}+1\right) M}+\frac{\epsilon}{p_{+} 2^{p_{+}+1}} \\
< & \frac{\epsilon}{2}+\frac{\epsilon}{4}+\frac{\epsilon}{4} \\
= & \epsilon .
\end{aligned}
$$

Therefore, $\left|f_{k}(\cdot)\right|^{p(\cdot)} \rightarrow|f(\cdot)|^{p(\cdot)}$ in measure.

Now define

$$
h_{k}(x)=2^{p_{+}-1}\left|f_{k}(x)\right|^{p(x)}+2^{p_{+}-1}|f(x)|^{p(x)}-\left|f(x)-f_{k}(x)\right|^{p(x)} \geq 0 ;
$$

then $h_{k} \rightarrow 2^{p_{+}}|f(\cdot)|^{p(\cdot)}$ in measure. Therefore, by Fatou's lemma on the classical Lebesgue spaces with respect to convergence in measure (see [5]),

$$
\begin{array}{r}
2^{p_{+}} \int_{\Omega}|f(x)|^{p(x)} d x \leq \liminf _{k \rightarrow \infty} \int_{\Omega} 2^{p_{+}-1}\left|f_{k}(x)\right|^{p(x)}+2^{p_{+}-1}|f(x)|^{p(x)} \\
-\left|f(x)-f_{k}(x)\right|^{p(x)} d x .
\end{array}
$$

Rearranging terms and using the fact that $\rho\left(f_{k}\right) \rightarrow \rho(f)$ we get that

$$
\limsup _{k \rightarrow \infty} \int_{\Omega}\left|f(x)-f_{k}(x)\right|^{p(x)} d x \leq 0 .
$$

Therefore, $f_{k} \rightarrow f$ in modular and our proof is complete.

\section{Proof of Theorem 1.3}

First suppose that $f_{k} \rightarrow f$ in norm. As shown in [3], for any exponent $p(\cdot)$, if $\|g\|_{p(\cdot)} \leq 1$, then $\rho(g) \leq\|g\|_{p(\cdot)}$. Therefore, for all $k$ sufficiently large,

$$
\rho\left(f-f_{k}\right) \leq\left\|f-f_{k}\right\|_{p(\cdot)} \leq 1 .
$$

Since the middle term goes to 0 as $k \rightarrow \infty$, it follows that $f_{k} \rightarrow f$ in modular. 
To prove the converse, note first that if $p_{-}=\infty$, then $\rho(g)=\|g\|_{L^{\infty}\left(\Omega_{\infty}\right)}=$ $\|g\|_{p(\cdot)}$, and so clearly convergence in norm and in modular are equivalent.

Now assume $p_{+}\left(\Omega \backslash \Omega_{\infty}\right)<\infty$. We know that there exists $\beta>0$ such that $\rho\left(\beta\left(f-f_{k}\right)\right) \rightarrow 0$. Fix $\lambda, 0<\lambda<\beta^{-1}$. Then

$$
\begin{aligned}
\rho\left(\left(f-f_{k}\right) / \lambda\right)= & \int_{\Omega \backslash \Omega_{\infty}}\left(\frac{\beta\left|f(x)-f_{k}(x)\right|}{\beta \lambda}\right)^{p(x)} d x \\
& +\frac{1}{\beta \lambda}\left\|\beta\left(f-f_{k}\right)\right\|_{L^{\infty}\left(\Omega_{\infty}\right)} \\
\leq & \left(\frac{1}{\beta \lambda}\right)^{p_{+}\left(\Omega \backslash \Omega_{\infty}\right)} \rho\left(\beta\left(f-f_{k}\right)\right) .
\end{aligned}
$$

Hence, for all $k$ sufficiently large we have that

$$
\rho\left(\frac{f-f_{k}}{\lambda}\right) \leq 1 .
$$

Equivalently, for all such $k,\left\|f-f_{k}\right\|_{p(\cdot)} \leq \lambda$. Since $\lambda$ can be arbitrarily small, $f_{k} \rightarrow f$ in norm.

Now suppose $p_{-}<\infty$ and $p_{+}\left(\Omega \backslash \Omega_{\infty}\right)=\infty$. We will construct a sequence $\left\{f_{k}\right\} \subset L^{p(\cdot)}(\Omega)$ such that $f_{k} \rightarrow 0$ in modular but $\left\|f_{k}\right\|_{p(\cdot)} \geq$ $1 / 2$ for all $k$. By the definition of the essential supremum, there exists a sequence of sets $\left\{E_{k}\right\}$ with finite measure such that:

(1) $E_{k} \subset \Omega \backslash \Omega_{\infty}$,

(2) $E_{k+1} \subset E_{k}$ and $\left|E_{k} \backslash E_{k+1}\right|>0$,

(3) $\left|E_{k}\right| \rightarrow 0$,

(4) if $x \in E_{k}, p(x) \geq p_{k}>k$.

Define the function $f$ by

$$
f(x)=\left(\sum_{k=1}^{\infty} \frac{1}{2^{k}\left|E_{k} \backslash E_{k+1}\right|} \chi_{E_{k} \backslash E_{k+1}}(x)\right)^{1 / p(x)},
$$

and for each $k$ let $f_{k}=f \chi_{E_{k}}$. Then for all $k \geq 1$,

$$
\rho\left(f_{k}\right)=\sum_{j=k}^{\infty} \int_{E_{j} \backslash E_{j+1}} \frac{1}{2^{j}\left|E_{j} \backslash E_{j+1}\right|} d x=\sum_{j=k}^{\infty} 2^{-j}=2^{-k+1} ;
$$


hence, $f_{k} \in L^{p(\cdot)}(\Omega)$ and $\rho\left(f_{k}\right) \rightarrow 0$ as $k \rightarrow \infty$. On the other hand, for all $k \geq 1$,

$$
\rho\left(\frac{f_{k}}{1 / 2}\right)=\sum_{j=k}^{\infty} \int_{E_{j} \backslash E_{j+1}} \frac{2^{p(x)}}{2^{j}\left|E_{j} \backslash E_{j+1}\right|} d x \geq \sum_{j=k}^{\infty} 2^{p_{j}-j}=\infty .
$$

Thus, $\left\|f_{k}\right\|_{p(\cdot)} \geq 1 / 2$.

\section{Proof of Theorem 1.4}

Suppose to the contrary that there exists a sequence $\left\{f_{k}\right\}$ that converges to $f$ in norm but not in measure. Then by passing to a subsequence we may assume that there exists $\epsilon, 0<\epsilon<1$, such that for all $k$,

$$
\left|\left\{x \in \Omega:\left|f(x)-f_{k}(x)\right| \geq \epsilon\right\}\right| \geq \epsilon .
$$

Denote the set on the left-hand side by $A_{k}$; since for each $k$ either $\mid A_{k} \cap$ $\Omega_{\infty} \mid \geq \epsilon / 2$ or $\left|A_{k} \backslash \Omega_{\infty}\right| \geq \epsilon / 2$, by passing to another subsequence we may assume that one of these inequalities holds for all $k$.

If $\left|A_{k} \cap \Omega_{\infty}\right| \geq \epsilon / 2$ for all $k$, then

$$
\left\|f-f_{k}\right\|_{p(\cdot)} \geq\left\|\left(f-f_{k}\right) \chi_{\Omega_{\infty}}\right\|_{p(\cdot)}=\left\|f-f_{k}\right\|_{L^{\infty}\left(\Omega_{\infty}\right)} \geq \epsilon,
$$

contradicting our assumption that $f_{k}$ converges to $f$ in norm. If $\mid A_{k} \backslash$ $\Omega_{\infty} \mid \geq \epsilon / 2$ for all $k$, then

$$
\begin{aligned}
\rho\left(\frac{f-f_{k}}{\epsilon^{2} / 2}\right) & \geq \int_{\Omega \backslash \Omega_{\infty}}\left(\frac{\left|f(x)-f_{k}(x)\right|}{\epsilon^{2} / 2}\right)^{p(x)} d x \\
& \geq \int_{A_{k} \backslash \Omega_{\infty}}\left(\frac{2}{\epsilon}\right)^{p(x)} d x \geq\left(\frac{2\left|A_{k} \backslash \Omega_{\infty}\right|}{\epsilon}\right) \geq 1 .
\end{aligned}
$$

Hence, $\left\|f-f_{k}\right\|_{p(\cdot)} \geq \epsilon^{2} / 2>0$, again contradicting our assumption that $f_{k}$ converges to $f$ in norm.

The proof that if $f_{k} \rightarrow f$ in norm, then for all $\gamma>0$ sufficiently small, $\rho\left(\gamma f_{k}\right) \rightarrow \rho(\gamma f)$, is very similar to but simpler than the proof if we only assume convergence in modular, and we therefore defer the proof until the end of the proof of Theorem 1.5 in Section 5 below.

Finally, we show that if $p_{+}=\infty$, then convergence in measure never implies convergence in norm. To do so we will construct a sequence $\left\{f_{k}\right\}$ that converges to 0 in measure but not in norm. If $\left|\Omega_{\infty}\right|>0$, then we have already done so in Example 1.7, since for functions supported on $\Omega_{\infty}$ the norm and the modular are equal. 
Now suppose $\left|\Omega_{\infty}\right|=0$ and $p_{+}\left(\Omega \backslash \Omega_{\infty}\right)=\infty$. Let the sets $E_{k}$ and the functions $f_{k}$ be defined as in the second half of the proof of Theorem 1.3 above. Then $\rho\left(f_{k}\right) \rightarrow 0=\rho(0)$, and since $\left|E_{k}\right| \rightarrow 0$, the functions $f_{k}$ converge to 0 in measure. On the other hand, $\left\|f_{k}\right\|_{p(\cdot)} \geq 1 / 2$ so the sequence does not converge in norm to 0 .

\section{Proof of Theorem 1.5}

We first assume that condition (2) holds. Let $f_{k} \rightarrow f$ in modular. As we noted above just after the statement of Theorem 1.4, if $\left|\Omega_{\infty}\right|>0$, it is straightforward to prove that $f_{k} \rightarrow f$ in measure on $\Omega_{\infty}$, so it would suffice to prove that we have convergence in measure on $\Omega \backslash \Omega_{\infty}$. Therefore, without loss of generality we will treat the special case when $\left|\Omega_{\infty}\right|=0$.

Since we have convergence in modular, there exists $\beta>0$ such that as $k \rightarrow 0$,

$$
\int_{\Omega}\left|\beta\left(f(x)-f_{k}(x)\right)\right|^{p(x)} d x \rightarrow 0 .
$$

Therefore, $\left|\beta\left(f(\cdot)-f_{k}(\cdot)\right)\right|^{p(\cdot)} \rightarrow 0$ in measure.

Recall that in our case, for each $M>1$,

$$
G_{M}=\{x \in \Omega: p(x)>M\} .
$$

By assumption, for any $\epsilon>0$, there exists $M_{0}>1$ such that if $M>M_{0}$, then $\left|G_{M}\right|<\epsilon / 2$. Fix $\epsilon, 0<\epsilon<1 / \beta$, and fix $M>M_{0}$ such that this is the case and such that $(\beta \epsilon)^{M}<\epsilon / 2$. Then for all $k$ sufficiently large,

$$
\begin{aligned}
\mid\{x \in \Omega: \mid f & \left.(x)-f_{k}(x) \mid>\epsilon\right\} \mid \\
& \leq\left|G_{M}\right|+\left|\left\{x \in \Omega \backslash G_{M}:\left|f(x)-f_{k}(x)\right|>\epsilon\right\}\right| \\
& \leq\left|G_{M}\right|+\left|\left\{x \in \Omega \backslash G_{M}:\left|\beta\left(f(x)-f_{k}(x)\right)\right|^{p(x)}>(\beta \epsilon)^{M}\right\}\right| \\
& <\frac{\epsilon}{2}+(\beta \epsilon)^{M} \\
& <\epsilon .
\end{aligned}
$$

Thus, $f_{k} \rightarrow f$ in measure.

We now show that for every $\gamma>0$ sufficiently small, $\rho\left(\gamma f_{k}\right) \rightarrow \rho(\gamma f)$. Since convergence in modular implies that $\left\|f-f_{k}\right\|_{L^{\infty}\left(\Omega_{\infty}\right)} \rightarrow 0$, by the triangle inequality we have that for any $\gamma>0,\left\|\gamma f_{k}\right\|_{L^{\infty}\left(\Omega_{\infty}\right)} \rightarrow$ $\|\gamma f\|_{L^{\infty}\left(\Omega_{\infty}\right)}$; therefore, as before we may restrict to the special case that $\left|\Omega_{\infty}\right|=0$. 
Because $f_{k} \rightarrow f$ in modular, there exists $\beta>0$ such that $\rho(\beta(f-$ $\left.\left.f_{k}\right)\right) \rightarrow 0$. We will first show that we can take $\gamma=\beta / 5$; since for every $\beta^{\prime}$, $0<\beta^{\prime}<\beta$, we have that $\rho\left(\beta^{\prime}\left(f-f_{k}\right)\right) \leq \rho\left(\beta\left(f-f_{k}\right)\right)$, it follows at once that we can take any $\gamma \leq \beta / 5$.

Since $f \in L^{p(\cdot)}(\Omega)$ and since we may replace $\beta$ by a smaller value, we can also assume that $\rho(\beta f)<\infty$. In particular, $|\beta f(x)|^{p(x)} d x$ is an absolutely continuous measure with respect to Lebesgue measure.

Fix $\epsilon>0$; since $\left|G_{M}\right| \rightarrow 0$ as $M \rightarrow \infty$, there exists $M>1$ such that $\rho_{G_{M}}(\beta f)<\epsilon$. Therefore, we have that

$$
\begin{aligned}
\rho_{G_{M}}\left(\frac{\beta}{2} f_{k}\right) & =\int_{G_{M}}\left(\frac{\beta}{2}\left|f_{k}(x)\right|\right)^{p(x)} d x \\
& \leq \int_{G_{M}} 2^{p(x)-1}\left[\left(\frac{\beta}{2}|f(x)|\right)^{p(x)}+\left(\frac{\beta}{2}\left|f(x)-f_{k}(x)\right|\right)^{p(x)}\right] d x \\
& \leq \frac{1}{2} \rho_{G_{M}}(\beta f)+\frac{1}{2} \rho_{G_{M}}\left(\beta\left(f-f_{k}\right)\right) \\
& <\frac{\epsilon}{2}+\frac{1}{2} \rho\left(\beta\left(f-f_{k}\right)\right) .
\end{aligned}
$$

Now let $\gamma=\beta / 5$. (The choice of 5 will become clear below.) Let $F_{M}=\Omega \backslash G_{M}$. Then

$$
\left|\rho(\gamma f)-\rho\left(\gamma f_{k}\right)\right| \leq\left|\rho_{G_{M}}(\gamma f)-\rho_{G_{M}}\left(\gamma f_{k}\right)\right|+\left|\rho_{F_{M}}(\gamma f)-\rho_{F_{M}}\left(\gamma f_{k}\right)\right| .
$$

To estimate the first term on the right-hand side, it suffices to note that $\left|\rho_{G_{M}}(\gamma f)-\rho_{G_{M}}\left(\gamma f_{k}\right)\right| \leq \frac{1}{5} \rho_{G_{M}}(\beta f)+\frac{\epsilon}{2}+\frac{1}{2} \rho\left(\beta\left(f-f_{k}\right)\right) \leq \epsilon+\frac{1}{2} \rho\left(\beta\left(f-f_{k}\right)\right)$.

To estimate the second term we argue as we did in the first part of the proof of Theorem 1.1:

$$
\begin{aligned}
& \left|\rho_{F_{M}}(\gamma f)-\rho_{F_{M}}\left(\gamma f_{k}\right)\right| \\
& \quad \leq \int_{F_{M}} p(x)\left(|\gamma f(x)|^{p(x)-1}+\left|\gamma f_{k}(x)\right|^{p(x)-1}\right)\left|\gamma\left(f(x)-f_{k}(x)\right)\right| d x \\
& \quad=\int_{F_{M} \cap \Omega_{1}}+\int_{F_{M} \cap \Omega_{*}} .
\end{aligned}
$$

The first integral is easy to bound since it immediately reduces to

$$
\int_{F_{M} \cap \Omega_{1}} 2\left|\gamma\left(f(x)-f_{k}(x)\right)\right| d x \leq \frac{2}{5} \rho\left(\beta\left(f-f_{k}\right)\right) .
$$


To estimate the second integral fix $\epsilon, 0<\epsilon<1 / 4$, and apply Young's inequality to get

$$
\begin{aligned}
\int_{F_{M} \cap \Omega_{*}} \epsilon^{p^{\prime}(x)} \frac{p(x)}{p^{\prime}(x)} & \left(|\gamma f(x)|^{p(x)-1}+\left|\gamma f_{k}(x)\right|^{p(x)-1}\right)^{p^{\prime}(x)} d x \\
+ & \int_{F_{M} \cap \Omega_{*}} \epsilon^{-p(x)}\left|\gamma\left(f(x)-f_{k}(x)\right)\right|^{p(x)} d x=I_{1}+I_{2} .
\end{aligned}
$$

We immediately have that

$$
I_{2} \leq \frac{\epsilon^{-M}}{5} \rho\left(\beta\left(f-f_{k}\right)\right) .
$$

To estimate $I_{1}$ we argue as before to get

$$
\begin{aligned}
I_{1} \leq & \int_{F_{M} \cap \Omega_{*}} \epsilon^{p^{\prime}(x)}(p(x)-1) \max \left(1,2^{2-p(x)}\right)^{p^{\prime}(x)}\left(|\gamma f(x)|+\left|\gamma f_{k}(x)\right|\right)^{p(x)} d x \\
\leq & \int_{F_{M} \cap \Omega_{*}} p(x)(4 \epsilon)^{p^{\prime}(x)}\left(2|\gamma f(x)|+\left|\gamma\left(f(x)-f_{k}(x)\right)\right|\right)^{p(x)} d x \\
\leq & 4 \epsilon \int_{F_{M} \cap \Omega_{*}} p(x) 2^{p(x)}\left(2^{p(x)}|\gamma f(x)|^{p(x)}+\left|\gamma\left(f(x)-f_{k}(x)\right)\right|^{p(x)}\right) d x \\
\leq & 4 \epsilon \int_{F_{M} \cap \Omega_{*}} p(x) 4^{p(x)}|\gamma f(x)|^{p(x)} d x \\
& +4 \epsilon \int_{F_{M} \cap \Omega_{*}} p(x) 2^{p(x)}\left|\gamma\left(f(x)-f_{k}(x)\right)\right|^{p(x)} d x \\
\leq & 8 \epsilon \rho(\beta f)+8 \epsilon \rho\left(\beta\left(f-f_{k}\right)\right) .
\end{aligned}
$$

To get the last inequality we use the fact that for all $x \in \Omega_{*}, p(x) 4^{p(x)} \leq$ $2 \cdot 5^{p(x)}$. (Here our choice of the value 5 above is used.)

Combining all of these estimates we get

$$
\left|\rho(\gamma f)-\rho\left(\gamma f_{k}\right)\right|<\epsilon+8 \epsilon \rho(\beta f)+\left(\frac{1}{2}+8 \epsilon+\frac{\epsilon^{-M}}{5}+\frac{2}{5}\right) \rho\left(\beta\left(f-f_{k}\right)\right) .
$$

Therefore, in the limit we have that

$$
\limsup _{k \rightarrow \infty}\left|\rho(\gamma f)-\rho\left(\gamma f_{k}\right)\right| \leq \epsilon+8 \epsilon \rho(\beta f) .
$$

Since $\epsilon>0$ was arbitrary, it follows that

$$
\lim _{k \rightarrow \infty}\left|\rho(\gamma f)-\rho\left(\gamma f_{k}\right)\right|=0 .
$$


To prove the converse implication, suppose to the contrary that condition (2) does not hold. We will construct a sequence that converges to 0 in modular but not in measure. By assumption, there exists $\delta>0$ such that for all $M>1,\left|G_{M}\right| \geq \delta$. Let $F_{M} \subset G_{M}$ be such that $\left|F_{M}\right|=\delta$ and define $f_{M}=\frac{1}{2} \chi_{F_{M}}$. Then

$$
\rho\left(f_{M}\right)=\int_{F_{M}} 2^{-p(x)} d x \leq 2^{-M} \delta .
$$

Therefore, $\rho\left(f_{M}\right) \rightarrow 0$ as $M \rightarrow \infty$; thus the sequence $\left\{f_{M}\right\}$ converges to 0 in modular. On the other hand, for any $\epsilon, 0<\epsilon<1 / 2$,

$$
\left|\left\{x \in \Omega:\left|f_{M}(x)\right| \geq \epsilon\right\}\right|=\left|F_{M}\right|=\delta,
$$

so this sequence does not converge to 0 in measure.

The proof for convergence in norm. We now complete the proof of Theorem 1.4. If $f_{k} \rightarrow f$ in norm, then we can modify the above proof to again show that $\rho\left(\gamma f_{k}\right) \rightarrow \rho(\gamma f)$ for all $\gamma>0$ sufficiently small. Since

$$
\left\|f-f_{k}\right\|_{L^{\infty}\left(\Omega_{\infty}\right)}=\left\|\left(f-f_{k}\right) \chi_{\Omega_{\infty}}\right\|_{p(\cdot)} \leq\left\|f-f_{k}\right\|_{p(\cdot)},
$$

by the triangle inequality we again have for all $\gamma>0$ that $\left\|\gamma f_{k}\right\|_{L^{\infty}\left(\Omega_{\infty}\right)} \rightarrow$ $\|\gamma f\|_{L^{\infty}\left(\Omega_{\infty}\right)}$, so we can again assume $\left|\Omega_{\infty}\right|=0$. Further, it will again suffice to prove that $\rho\left(\gamma f_{k}\right) \rightarrow \rho(\gamma f)$ for some $\gamma>0$.

Fix $\beta>0$ such that $\rho(\beta f)<\infty$. Then arguing as we did to prove (3.1), we have that for all $k$ sufficiently large,

$$
\rho\left(\beta\left(f-f_{k}\right)\right) \leq\left\|\beta\left(f-f_{k}\right)\right\|_{p(\cdot)} \leq 1 .
$$

In particular, $\rho\left(\beta\left(f-f_{k}\right)\right) \rightarrow 0$.

Now let $\gamma=\beta$. Then the argument is very similar to the previous, except that we do not need to consider the sets $G_{M}$ and $F_{M}$. Instead we estimate as follows:

$$
\begin{aligned}
& \left|\rho(\gamma f)-\rho\left(\gamma f_{k}\right)\right| \\
\leq & \int_{\Omega} p(x)\left(|\gamma f(x)|^{p(x)-1}+\left|\gamma f_{k}(x)\right|^{p(x)-1}\left|\gamma\left(f(x)-f_{k}(x)\right)\right| d x=\int_{\Omega_{1}}+\int_{\Omega_{*}} .\right.
\end{aligned}
$$

We estimate the first integral exactly as before. To estimate the second integral we again apply Young's inequality to get two integrals $I_{1}$ and $I_{2}$. The estimate for $I_{1}$ goes through as before with no change except replacing the domain of integration $F_{M} \cap \Omega_{*}$ by $\Omega_{*}$. To estimate $I_{2}$, it suffices to note that again invoking the result from $[\mathbf{3}]$, for all $k$ sufficiently large, 
by $(5.1)$

$I_{2}=\int_{\Omega_{*}} \epsilon^{-p(x)}\left|\gamma\left(f(x)-f_{k}(x)\right)\right|^{p(x)} \leq \rho\left(\epsilon^{-1} \gamma\left|f-f_{k}\right|\right) \leq \epsilon^{-1} \gamma\left\|f-f_{k}\right\|_{p(\cdot)}$, and so $I_{2} \rightarrow 0$ as $k \rightarrow \infty$. The remainder of the argument now goes through without change.

\section{Proof of Theorem 1.8 and Example 1.9}

Proof of Theorem 1.8. We prove this by modifying the second half of the proof of Theorem 1.1. Since $f_{k} \rightarrow f$ in measure, for any $\epsilon$ and $\alpha$, $0<\epsilon<1,0<\alpha<1$, we have that for all $k$ sufficiently large,

$$
\begin{aligned}
\mid\{x \in \Omega: \mid \alpha( & \left.\left.f(x)-f_{k}(x)\right)\left.\right|^{p(x)}>\epsilon\right\} \mid \\
& \leq\left|\left\{x \in \Omega:\left|f(x)-f_{k}(x)\right|>\alpha^{-1} \epsilon^{1 / p_{-}}\right\}\right| \\
& \leq\left|\left\{x \in \Omega:\left|f(x)-f_{k}(x)\right|>\epsilon\right\}\right| \\
& <\epsilon .
\end{aligned}
$$

Hence,

$$
\left|\alpha\left(f(\cdot)-f_{k}(\cdot)\right)\right|^{p(\cdot)} \rightarrow 0
$$

in measure.

Fix $\gamma>0$ as in the hypotheses, and let $\beta=\gamma / 3<1$. Then we have that $\rho(\gamma f)<\infty$ and $\rho\left(\beta f_{k}\right) \rightarrow \rho(\beta f)$. Furthermore, for almost every $x \in \Omega, p(x)\left(2^{p(x)-1}+1\right) \leq 3^{p(x)}$, and so by (2.1) and (2.2),

$$
\begin{aligned}
|| \beta f(x) & \left.\right|^{p(x)}-\left|\beta f_{k}(x)\right|^{p(x)} \mid \\
\leq & p(x)\left(|\beta f(x)|^{p(x)-1}+\left|\beta f_{k}(x)\right|^{p(x)-1}\right)\left|\beta f(x)-\beta f_{k}(x)\right| \\
\leq & p(x)|\beta f(x)|^{p(x)-1}\left|\beta f(x)-\beta f_{k}(x)\right| \\
& +p(x) \max \left(1,2^{p(x)-2}\right) \\
& \times\left(|\beta f(x)|^{p(x)-1}+\left|\beta f(x)-\beta f_{k}(x)\right|^{p(x)-1}\right)\left|\beta f(x)-\beta f_{k}(x)\right| \\
\leq & p(x)\left(2^{p(x)-1}+1\right)|\beta f(x)|^{p(x)-1}\left|\beta\left(f(x)-f_{k}(x)\right)\right| \\
& +p(x) 2^{p(x)-1}\left|\beta\left(f(x)-f_{k}(x)\right)\right|^{p(x)} \\
\leq & |\gamma f(x)|^{p(x)-1}\left|\gamma\left(f(x)-f_{k}(x)\right)\right|+\left|\gamma\left(f(x)-f_{k}(x)\right)\right|^{p(x)} .
\end{aligned}
$$


Fix $\epsilon>0$. Let

$$
H_{M}=\left\{x \in \Omega:|\gamma f(x)|^{p(x)-1}>M\right\}
$$

since $\rho(\gamma f)<\infty,|\gamma f(\cdot)|^{p(\cdot)} \in L^{1}(\Omega)$, and so there exists $M \geq 1$ such that $\left|H_{M}\right| \leq \epsilon / 4$. Hence,

$$
\begin{aligned}
\mid\{x \in \Omega:|| \beta f(x) & \left.\left.\right|^{p(x)}-\left|\beta f_{k}(x)\right|^{p(x)} \mid>\epsilon\right\} \mid \\
\leq & \left|H_{M}\right| \\
& +\left|\left\{x \in \Omega \backslash H_{M}: M\left|\gamma\left(f(x)-f_{k}(x)\right)\right|>3 \epsilon / 4\right\}\right| \\
& +\left|\left\{x \in \Omega \backslash H_{M}:\left|\gamma\left(f(x)-f_{k}(x)\right)\right|^{p(x)}>\epsilon / 4\right\}\right| \\
\leq & \left|H_{M}\right| \\
& +\left|\left\{x \in \Omega \backslash H_{M}:\left|\beta\left(f(x)-f_{k}(x)\right)\right|>\epsilon / 4 M\right\}\right| \\
& +\left|\left\{x \in \Omega \backslash H_{M}:\left|\gamma\left(f(x)-f_{k}(x)\right)\right|^{p(x)}>\epsilon / 4\right\}\right| \\
< & \frac{\epsilon}{4}+\frac{\epsilon}{4 M}+\frac{\epsilon}{4} \\
< & \epsilon
\end{aligned}
$$

the second to last inequality holds by our choice of $M$, since $\beta f_{k} \rightarrow$ $\beta f$ in measure, and by inequality (6.1) with $\alpha=\gamma<1$. Therefore, $\left|\beta f_{k}(\cdot)\right|^{p(\cdot)} \rightarrow|\beta f(\cdot)|^{p(\cdot)}$ in measure.

Now let $\bar{\beta}=\beta / 2$ and define

$$
h_{k}(x)=\left|\beta f_{k}(x)\right|^{p(x)}+|\beta f(x)|^{p(x)}-\left|\bar{\beta}\left(f(x)-f_{k}(x)\right)\right|^{p(x)} .
$$

Since

$$
\left|\bar{\beta}\left(f(x)-f_{k}(x)\right)\right|^{p(x)} \leq 2^{p(x)-1}\left(|\bar{\beta} f(x)|^{p(x)}+\left|\bar{\beta} f_{k}(x)\right|^{p(x)}\right),
$$

$h_{k}(x) \geq 0$. Further, by (6.1) with $\alpha=\bar{\beta}, h_{k} \rightarrow 2|\beta f(\cdot)|^{p(\cdot)}$ in measure. Therefore, by Fatou's lemma with respect to convergence in measure,

$$
\begin{aligned}
& 2 \int_{\Omega}|\beta f(x)|^{p(x)} d x \\
& \quad \leq \liminf _{k \rightarrow \infty} \int_{\Omega}|\beta f(x)|^{p(x)}+\left|\beta f_{k}(x)\right|^{p(x)}-\left|\bar{\beta}\left(f(x)-f_{k}(x)\right)\right|^{p(x)} d x .
\end{aligned}
$$

Re-arranging terms and using the fact that $\rho\left(\beta f_{k}\right) \rightarrow \rho(\beta f)$, we have that

$$
\limsup _{k \rightarrow 0} \int_{\Omega}\left|\bar{\beta}\left(f(x)-f_{k}(x)\right)\right|^{p(x)} d x \leq 0 .
$$

Hence, $f_{k} \rightarrow f$ in modular. 
Construction of Example 1.9. Fix a set $E \subset \Omega$ such that $|E|<\infty$ and $p_{+}(E)<\infty$. Let $\left\{E_{k}\right\}$ be a sequence of subsets of $E$ of positive measure such that $\left|E_{k}\right| \rightarrow 0$. Define $f=\chi_{E}$ and

$$
f_{k}=\chi_{E \backslash E_{k}}+\left|E_{k}\right|^{-2} \chi_{E_{k}} .
$$

Since $\left|E_{k}\right| \rightarrow 0, f_{k} \rightarrow f$ in measure. For any $\gamma, 0<\gamma<1$,

$$
\rho(\gamma f)=\int_{E} \gamma^{p(x)} d x<|E|,
$$

and, since for all $k$ sufficiently large, $\left|E_{k}\right|^{2}<\gamma$,

$$
\begin{aligned}
\rho\left(\gamma f_{k}\right)= & \int_{E \backslash E_{k}} \gamma^{p(x)} d x \\
& +\int_{E_{k}}\left(\gamma\left|E_{k}\right|^{-2}\right)^{p(x)} d x<|E|+\left|E_{k}\right|\left(\gamma\left|E_{k}\right|^{-2}\right)^{p_{+}(E)}<\infty .
\end{aligned}
$$

Hence, $\left\{f_{k}\right\} \subset L^{p(\cdot)}(\Omega)$ and $f \in L^{p(\cdot)}(\Omega)$. On the other hand, for all such values of $k$,

$$
\rho\left(\gamma f_{k}\right) \geq \int_{E_{k}}\left(\gamma\left|E_{k}\right|^{-2}\right)^{p(x)} d x \geq \gamma\left|E_{k}\right|^{-1} .
$$

Thus, $\rho\left(\gamma f_{k}\right) \rightarrow \infty$ and so does not converge to $\rho(\gamma f)$.

Similarly, given any $\beta>0$, for all $k$ sufficiently large we have that

$$
\beta\left(\left|E_{k}\right|^{-2}-1\right) \geq 1
$$

and so

$$
\rho\left(\beta\left(f-f_{k}\right)\right) \geq\left|E_{k}\right| \beta\left(\left|E_{k}\right|^{-2}-1\right) .
$$

Since the right-hand side diverges, $f_{k}$ does not converge to $f$ in modular.

\section{References}

[1] L. Diening, P. Hästö, And A. Nekvinda. Open problems in variable exponent Lebesgue and Sobolev spaces, in: "Function Spaces, Differential Operators and Nonlinear Analysis Proceedings" (FSDONA 2004), Academy of Sciences of the Czech Republic, Prague, 2005 , pp. 38-52.

[2] X. FAn AND D. ZhaO, On the spaces $L^{p(x)}(\Omega)$ and $W^{m, p(x)}(\Omega), J$. Math. Anal. Appl. 263(2) (2001), 424-446.

[3] O. KovÁČIK AND J. RÁKosník, On spaces $L^{p(x)}$ and $W^{k, p(x)}$, Czechoslovak Math. J. 41(116), no. 4 (1991), 592-618.

[4] W. OrLicz, Über konjugierte Exponentenfolgen, Studia Math. 3 (1931), 200-211. 
[5] H. L. Royden, "Real analysis", Third edition, Macmillan Publishing Company, New York, 1988.

[6] S. SAmkO, On a progress in the theory of Lebesgue spaces with variable exponent: maximal and singular operators, Integral Transforms Spec. Funct. 16(5-6) (2005), 461-482.

David Cruz-Uribe, SFO:

Department of Mathematics

Trinity College

Hartford, CT 06106-3100

USA

E-mail address: david.cruzuribe@trincoll.edu

Alberto Fiorenza:

Dipartimento di Costruzioni e Metodi Matematici in Architettura

Università di Napoli

Via Monteoliveto, 3

I-80134 Napoli

Italy

and

Istituto per le Applicazioni del Calcolo "Mauro Picone", sezione di Napoli

Consiglio Nazionale delle Ricerche

Via Pietro Castellino, 111

I-80131 Napoli

Italy

E-mail address: fiorenza@unina.it

Primera versió rebuda el 8 de juliol de 2009, darrera versió rebuda el 25 de gener de 2010. 